\title{
LAPUDUTM
}

Euskal ikerketen aldizkaria | Revue d'études basques |

Revista de estudios vascos | Basque studies review

2| 1997

Numéro II

\section{Le magicien-guérisseur du carnet de voyage de 1835 d'Antoine d'Abbadie}

\section{Yvette Cardaillac-Hermosilla}

\section{OpenEdition}

\section{Journals}

Édition électronique

URL : http://journals.openedition.org/lapurdum/1792

DOI : 10.4000/lapurdum.1792

ISSN : 1965-0655

\section{Éditeur}

IKER

Édition imprimée

Date de publication : 1 octobre 1997

Pagination : $93-107$

ISBN : 2-84127-142-0

ISSN : $1273-3830$

Référence électronique

Yvette Cardaillac-Hermosilla, « Le magicien-guérisseur du carnet de voyage de 1835 d'Antoine d'Abbadie », Lapurdum [En ligne], 2 | 1997, mis en ligne le 01 septembre 2010, consulté le 31 janvier 2020. URL : http://journals.openedition.org/lapurdum/1792 ; DOI : 10.4000/lapurdum.1792 
Yvette CARDAILLAC-HERMOSILLA

\section{LE MAGICIEN-GUÉRISSEUR DU CARNET DE VOYAGE DE 1835 D'ANTOINE D'ABBADIE}

Les éléments orientaux et magiques structurent le journal de voyage de 1935 écrit par Antoine d'Abbadie, âgé de 25 ans, à la recherche d'orientations en train de construire un destin marqué par les étoiles selon la conception astrologique et astronomique qui hantera toute l'époque classique et le Moyen Age avant de se dissocier et de s'opposer à la Renaissance. Le jeune homme ne va pas se démarquer de cette notion originelle puisqu'il va vivre tout au long de sa vie selon cette double orientation, c cest-à-dire la connaissance scientifique du monde stellaire comme l'a bien montré dans sa conférence lors du centenaire Agustin Sánchez Lavega' et la perspective symbolique, poétique et religieuse des étoiles, comme nous avons essayé de l'expliquer dans notre exposé « L'orientalisme au château d'Abbadie " ${ }^{2}$. Ainsi, au folio 15, apparaissent les notes sur son horoscope, situées environ au $2 / 9^{c}$ du texte, comprenant 73 folios. De même, $2 / 9^{\circ}$ avant la fin, au folio 53 , nous relevons le mot "Quiromancier » qui nous semble-t-il doit être lu " chiromancien ou chiromancie", en tenant compte des problèmes orthographiques répétés de ce basque français anglophone apprenant l'arabe. Il s'agit du titre d'un livre à relier qui montrerait l'intérêt concemant les diverses aspects des arts magiques ou divinatoires. En particulier la chiromancie, très pratiquée au XVI' siècle et au début du $X V I^{c}$, retrouve une grande vogue au XIX' siècle. Le capitaine d'Arpentigny et Desbarolles publient des ouvrages traitant à la fois de chiromancie ${ }^{3}$ et de chirognomonie ${ }^{4}$. Sur la même page $e^{5}$ nous trouvons une poésie arabe. Il présente le Cheikh, personnage clef de la poésie orientale, sublimé ici et nimbé d'une lumière mythique à la Delacroix ${ }^{5}$. Mais il recherche la femme fleur, l'amour dans une oasis ou le vent du désert. Ils apparaissent comme des stéréotypes qui nourrissent l'imaginaire de la bourgeoisie éclairée et de la noblesse du $\mathrm{XIX}^{*}$ siècle dans sa représentatiọn d'un Orient mythique. Rêve fugace d'un jeune homme de 25 ans, il relève des images et de l'échange timide qui caractérise l'attitude du magicien-guérisseur qui montre bien la communauté de construction entre les deux textes. Le portrait du magicien situé au dernier $9^{c}$ du texte par sa position apparaît comme le développement et le point d'orgue des thèmes magiques et orientalistes struc-

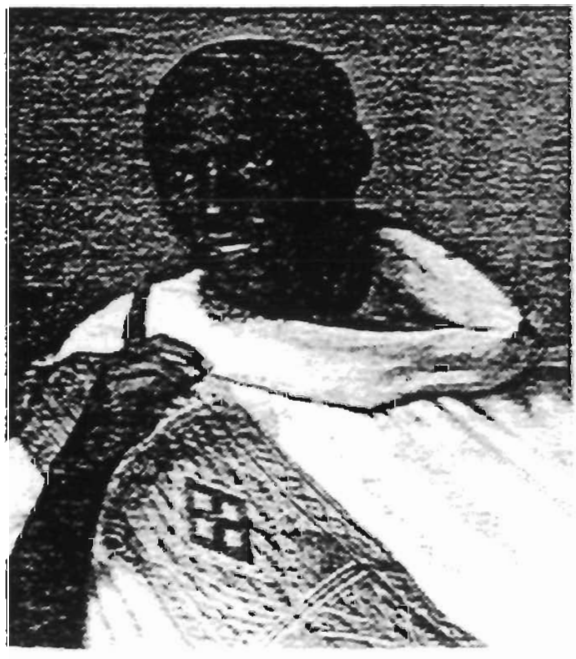

Portrait de chef éthiopien (rolé) châte au d'Abbadia 
turant le volume qui finit sur une note intéressante qui nous donne une possibilité d'analyse, le livre lui-même est considéré comme un premier-né, un fils chéri résultant du travail de l'écriture, écho du fils esclave du magicien et d' Antoine d'Abbadie, fils orphelin de père en 1835 .

Ces textes se répondent en écho dans le recueil et semblent former un tout car ils font référence à des intérêts et des connaissances complémentaires de leur auteur. Le portrait du magicien guérisseur qui voyage en Orient figurant sur le carnet de voyage d'Antoine d'Abbadie' en 1835 se situe du folio 65 verso au folio 66 recto, c'est-à-dire dans la partie finale. Selon son auteur, résultat d'un travail fait avec amour (“j”ai chéri", "caresses") et opiniâtreté ("corrections") comme préparation au voyage en Orient. dont rêvaient les jeunes gens bien nés et fortunés de l'époque romantique.

L'archétype du magicien est présent ici avec les instruments de son pouvoir, charmes, talismans, amulettes, soins, fabrication de l'or". Une technique de la narration individualise le personnage. Un syncrétisme des influences orientales et de l'attitude romantique mêle des références autobiographiques à une problématique aux limites flottantes entre magie, science et religion qu'il résout à sa manière (science, astuce/ignorance, foi).

L'archétype du magicien guérisseur présenté ici correspond à un modèle éternel qui se reproduit à travers le temps et l'espace avec des éléments fixes dont quelques aspects varient en fonction des circonstances historiques et des modalités géographiques. Ici, les attributs de son pouvoir sont les amulettes, talismans et charmes. Antoine d'Abbadie en fait des mots synonymes mais en réalité les spécialistes les différencient. Le talisman du persan « tilismat » vient du mot grec byzantin "cérémonie religieuse' et du grec 'faire un sacrifice'. C'est un mot d'origine savante d'importation européenne. Il apparaît en français en 1637. Les musulmans composent des talismans avec l'inscription sur une feuille ou un parchemin de versets du Coran et en particulier celles désignées sous le nom de Kousi (le trône). On les porte habituellement dans une boîte en or ou en argent suspendue au cou ou aux vêtements. Les amulettes se forment avec des plantes, du métal ou des pierres précieuses, souvent réunies dans des sachets de tissu précieux, elles apparaissent comme de second ordre par rappor aux talismans religieux. En Espagne, les nouveaux chrétiens ou musulmans convertis se caractérisent aux yeux des chrétiens par le port de talismans et cela même si les chrétiens eux-mêmes s'adonnent assez souvent à cette pratique. Dans le texte les charmes sont constituées par des inscriptions latines indiquant la place, la santé et les projets du magicien. Les instruments du pouvoir sont des talismans et le mot amulette conviendrait. Les boussoles et la montre sont présentées comme grandes amulettes avec un emploi convenable.

Dans ce portrait qui se veut noble et majestueux, la précision n'est pas le problème, au contraire l'écrivain crée toute une esthétique du flou destiné à donner du relief aux éléments indiscutables et essentiels. Le narrateur omniscient est le maître du pouvoir que lui confere l'écriture comme celui que le Verbe donne au magicien.

Dans cette fiction, les activités curatives se limitent aux pratiques courantes de soin en milieu rural mais elles n'abordent jamais les réalisations maléfiques des sorcières telles qu elles se sont développées dans les fantasmes populaires succédant à ceux des clercs au XVI' et XVII' siècles en Europe ${ }^{10}$. Le développement juridique de divers cas dans le village de Zugarramurdi, peu éloigné d'Hendaye et sans doute connu par le très cultivé Antoine d'Abbadie, ne s' intègre pas dans sa vision du monde. Par sa création il nous montre qu'il partage l'attitude éclairée de l'inquisiteur espagnol Salazar y 
Frías" qui, à Logroño, n'ajouta pas foi aux dires des enfants et des vieillards, témoins, croyaient-ils. de terribles manifestations. $D^{\prime}$ ailleurs comme le montrent les portraits de magiciens mis en annexes, le guérisseur en Orient fuit toute magie diabolique ou maléfique. Les concepts magiques précis peuvent être intervertis sans nuire à la fresque d'ensemble. Et l'essentiel est de brosser une scène de genre non à la manière de la peinture hollandaise réaliste du XVII' mais selon la mode régnant au XIX' siècle.

Les guérisons s'effectuent au moyen de produits simples "écorces de fruits secs et pulvérisés" et "substances innocentes". Bien que les indications ne soient pas très précises elles s intègrent dans la tradition de la médecine naturelle à base de simples utilisées dans les civilisations agraires. La relation avec l'au-delà est présentée lors de la consultation de ses boussoles ou de sa montre : pour le vulgaire il s'agit de se mettre en relation avec l'âme du père ou avec le bon génie/ange gardien. La fabrication de l'or à partir de métal vulgaire fait référence au rêve des alchimistes relié à la position des étoiles "il disait ne pouvoir en faire que sous certains aspects des astres" nous dit le narrateur.

Le point de vue de ce dernier, facteur essentiel de la mise en scène de la fiction détermine par son regard donne une vision qui hiérarchise, dirige et fixe les significations. Ici, il est omniscient car il dévoile les mécanismes de fonctionnement du personnage :

- « Sa boussole... était avec sa montre... ses grandes amulettes », il apparaît que le objets fruits de la science semblent des amulettes à ceux qui en ignorent le fonctionnement.

- « Ses charmes étaient des inscriptions latines indiquant sa place, sa santé, ses projets », enfin les talismans du magicien sont représentés concrètement par des pages similaires à celles du carnet de voyage mais écrites en latin, là aussi c'est l'ignorance de cette langue qui permet d'ajouter foi aux pouvoirs de ces inscriptions. Ce sont donc les connaissances scientifiques qui donnent le pouvoir sur des populations de culture agraire et traditionnelle qui correspondent tout à fait à l'optique colonisatrice du $\mathrm{XIX}^{\mathrm{c}}$ siècle. Cette politique crée cet Orient mythique considéré comme inférieur à I'Occident détenteur de la connaissance.

Le flou volontaire des notions de religion, magie et science projette certains aspects par contraste qui se détachent avec la plus grande netteté :

- «Tous les soirs lui et son fils, à deux genoux, priaient le Dieu des chrétiens et la Vierge Marie. »

- "Il se disait partout un basque, petite nation indépendante entre France et Espagne », la religion chrétienne, la nationalité basque sont les éléments centraux de la personnalité de ce magicien ambigu qui dans ses agissements mêle fiction et réalité pour entretenir le prestige qui lui permetra par ses activités de survivre lors de ses missions scientifiques.

Ce portrait se caractérise par le contraste entre le réel et l'imaginaire. L'absence de description du visage, lieu de l'affectivité et de la représentation de l'âme, ou du corps laissent place à une silhouette : « il porait l'habit de chaque pays mais beau et distingué ". Le narrateur nous parle de son air d'une gravité obstinée. La relation sociale est extrêmement importante, le magicien chercheur garde une attitude de maître et dans sa relation avec les autres son fils sert d'intermédiaire : "comme un esclave ". Lui ne traite qu' avec les marabouts c'eșt-à-dire les saints hommes qu'il traite d'égal à égal ou avec les chefs qu'il récompense à l'occasion de façon astucieuse 
pour se valoriser avec de l'or caché dans les ruines. L'utilisation de la parole distancée et noble, parler de soi à la troisième personne est exactement ce que fait l'écrivain dans ce texte qui lui sert de miroir où il essaie de lire le futur en employant le passé. A côté de ce rôle social le portrait moral nous campe un homme plein de ténacité qui « reste debout toute la joumée sans manger si nécessaire » pour montrer qu'il respecte l'engagement pris. Malgré l'attitude hautaine de maître et de chef, le voyageur garde son humilité religieuse en priant à deux genoux, sorte d'intermédiaire privilégié entre Dieu et les hommes peuplant l'Orient montrant ainsi un sens aigu de son rôle dans le monde qui ne lui laisse pas oublier une conscience encore plus profonde des rapports reposant sur l'argent et les échanges. Le paiement, les récompenses, les demandes ponctuent le texte comme un leitmotiv. Il caractérise le personnage de fiction : les rapports à I'argent se répètent de façon presque obsessionnelle :

- «Il se faisait payer de tous à moins que le malade ne déclarât qu'il n'avait pas de quoi »

- «Il portait six mille francs en or »... « il avait projet de cacher quelques pièces de temps en temps dans les ruines et de les y faire prendre par un chef auquel il aurait besoin de donner quelque récompense »

- «Quand il y avait une surabondance d'objets de valeur inférieure, il les faisait échanger par son fils contre chameaux, chevaux, couvertures de laine... »

- «Quand il n' avait pas reçu assez, i] ne se faisait pas scrupule de demander... »

- «S'il avait gagné assez d'argent il faisait tuer un ou deux moutons ou bœuf et les faisait distribuer aux pauvres »

Ces cinq références relativement abondantes dans un texte court font écho à d'autres créations de magiciens dans la fiction littéraire mais aussi à la réalité des pro-

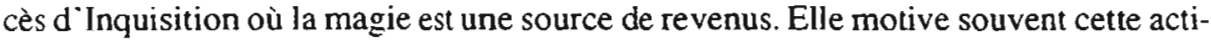
vité. Les notations d'intendance ou de prix peuvent être relevées tout au long du carnet de voyage ${ }^{12}$. Les problèmes financiers semblent au centre des intérêts d'Antoine d'Abbadie aussi bien dans la réalité que dans la fiction, l'achat de nombreux hectares à Hendaye et le financement de ses expéditions doivent être la cause essentielle d'une certaine dureté dans les rapports financiers. Mais c'est aussi un moyen de cultiver son prestige par l'astuce du trésor découvert, de pratiquer la charité envers les pauvres lorsqu' on se trouve dans l'abondance. Mais ces comptes de caractère systématique correspondent certes à une attitude scientifique et méthodique d'un administrateur comme d'ailleurs l'ordre systématique pour ranger les instruments scientifiques dans le coffre du magicien. Cependant, il laisse à son fils ou esclave ${ }^{13}$ les rôles secondaires ou peu prestigieux comme les échanges grâce aux aiguilles qui ne lui apporteraient ni honneur ni prestige. Mais dans la tradition de la charité orientale, un des piliers de l'Islam, il veut bien partager l'excès de bénéfice avec les pauvres par des sacrifices d'animaux se coulant dans la tradition comme le sacrifice du mouton coutumier pour la fête de l'Aìd El Khebir.

Les romantiques se contentent d'une appréciation imaginaire lorsqu'ils traitent le thème de l'Orient mythique, Victor Hugo dans Les Orientales (1829) utilise ses souvenirs enfantins d'une Espagne lumineuse pour peindre la Grèce et Musset décrit Venise la Rouge sans l'avoir encore jamais vue. Cet intérêt pour l'Orient. généré en particulier par la traduction des Mille et Une nuits par Galland au XVII' ${ }^{\circ}$ siècle provoque des fantaisies fragiles dont on a conscience. Musset n'écrit-il pas: 
«Si d'un coup de pinceau je vous avais bâti

quelques villes au toit bleu, quelques blanches mosquées,

quelques tirades en vers d'or et d'argent plaquées,

m'auriez-vous répondu : vous en avez menti ? ' $^{14}$

L'attitude du poète romantique s'accompagne de tout un courant de pensée surgi du bon sens populaire et qui convient au magicien-chercheur dans un pays d'Orient :

«A beau mentir qui vient de Ioin ", il est pris au sérieux, et « Nul n'est prophète en son pays $»$.

La vogue de l'exotisme"s au XIX" siècle concerne les productions des pays étrangers et lointains et génère la production de tableaux colorés qui montrent des moeurs différentes des nôtres.

La problématique posée par le texte laisse une volontaire imbrication des notions de magie, de science et de religion au profit de la création d'une auréole de mystère et de prestige autour du personnage, d'ailleurs teintée d'exagération volontaire :

"Ces instruments d'astronomie servaient à voir dans son pays... Si eux voulaient aller dans son pays il leur ferait voir leurs tentes ». (Science/magie)

- «(II) faisait toutes ses préparations, prières et incantations pour fabriquer des talismans en secret. (religion/magie).

Cependant il est clair que le narrateur omniscient qui révèle les mécanismes de fonctionnement de ce système différencie parfaitement les connaissances scientifiques et la magie. Tout ce qui permet une explication rationnelle s'exclut de cette dernière notion. Au contraire, les effets obtenus sous l'influence de l'expérience et de l'intuition peuvent en dépendre. Il est clair que les progrès dans le domaine des diverses sciences réduisent le champ d'action de la magie. Mais à la fois l'intégration complète de l'homme dans la société de consommation, dans la ville, ont tendance à lui faire oublier tout un savoir traditionnel nécessaire à l'équilibre du groupe social. Par ailleurs, religion et magie sont en concurrence dans les sociétés traditionnelles dans un système théocratique, les valeurs religieuses et les règles morales imposées par le pouvoir tendent au rejet de la magie que l'on craint lorsque son influence dépasse le cadre familial de la vie quotidienne. Cependant le regard de l'autre est fondamental pour la distinction entre ces deux notions. Pour l'oriental les formules latines par le mystère qu'elles représentent peuvent avoir un caractère magique.

Le travail conscient de réflexion sur l'écriture par le jeu des personnes, par la mise en abyme (note 28) s'apparente à des recherches déjà entreprises par Cervantés. En outre les écrivains réalistes et parnassiens ont introduit en littérature un besoin d'exactitude pour ainsi dire scientifique qui avait manqué jusqu'alors. Cependant Barthes montre comment le réalisme dans la description-portrait est lui-même une copie au second degré du réel, la reproduction par le langage d'une image picturale du monde conventionnelle et socialisée. L autoportrait et l"auto-mise en scène sont des genres narcissiques dans l'art de l'écriture ou de la peinture. L'écrivain s'affirme comme individu créateur depuis la philosophie humaniste et cette tendance se développe à l'époque romantique. Ces techniques posent le problème de l'image projetée de soi, de la représentation de soi pour les autres. Dans son procédé même la mise en abyme du geste, de l'attitude (je me dépeins en me décrivant) renvoie sans fin de la cause à l'effet, à la mort (mort du père, mort anticipée de soi-même), à l'aliénation. au vertige narcissique qui ici devient féroce et presque caricatural dans le rapport aux gains ou aux dons. rappelant des autoportraits célèbres comme ceux de Goya ou de Van Gogh. 


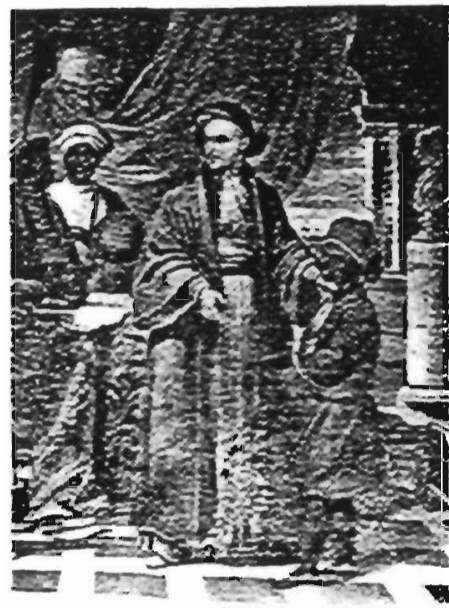

Portrail d Antoine d'Abbadie en costume éthiopien accompagné de son esclase Ahdirlah (volé)
L'image de soi dans ce type de production est socialement reconstituée à travers les codes culturels dominants en conformité avec un modele intériorisé. Il est intéressant de constater sa permanence puisque la silhouette, l'allure, l'image donnée du corps dans le texte correspond à celle du tableau commandé puis volé de Monsieur d'Abbadie en toge d'Ethiopien et turban accompagné de l'enfant esclave Abdullah, image d'un fils qu'il n'aura pas. Mais si dans le tableau les silhouettes sont statiques selon la tradition du portrait en pied, dans le texte nous avons des silhouettes agissantes jeunes et dynamiques, un portrait en mouvement, en voyage, le voyage de la vie, symbolique et réel, centré sur la magie de l'Orient mythique puis réel et enfin recréé des années plus tard au château d'Abbadia.

\section{Annexe 1}

Notes sur mon horoscope $\mathrm{e}^{16}$

Changement dans la $25^{\circ}$ année... Voyage en septembre, octobre, même changement encore. Un voyage : bon concernant des biens à vendre, à acheter mars 1835 . Mauvais temps pour la vie... Peut-être v... 1839. Mort d'une tante malade.

En 1840, maladie d'une amie au commencement de $1841 \ldots$ mort d'une sœur ou d'un parent.

En 1837, capricome dans mon ascendant De juin 1835 jusqu'à juillet 1836, bon terme pour amis et fort succès car capricorne traverse la onzième maison en septembre ou octobre. Voyage probable à Alger..

1841, désappointé en amour.

L'horoscope est en nocturne... signifie mon père...

1832 , mort de mon père..

\section{Annexe 2}

Folio 53 , p. $105^{17}$

... Donner à relier à Vogel : Quiromancier 1 vol. Moore 4. Rozet 3. Tom Cringle 1. Robray 1.

\section{Poésie arabe}

Dans les jardins de Kaïpha' ${ }^{18}$ il y a une fleur (folio 53 v) que le soleil cherche à travers les feuilles du palmier.

Cette fleur a des yeux plus doux que la gazelle, des yeux qui ressemble à une goutte d'eau de mer dans un coquillage.

Cette fleur a des parfums si enivrants que le Chekh qui s'enfuit devant la lance d'une autre tribu sur sa jument plus rapide que la chute des eaux, la sent au passage et s'arrête pour la respirer. 
Le vent du Simoun ${ }^{19}$ enlève des habits des voyageurs tous les autres parfums mais il n'enlève jamais du cœur l'odeur de cette fleur merveilleuse.

On la trouve au bord d' une source qui coule sans murmure à ses pieds.

Jeune fille. dis-moi le nom de ton père et je te dirai le nom de cette fleur.

\section{Annexe 3}

\section{La magicien-explorateur du carnet de voyage de 1835 d'Antoine d'Abbadie et son fils}

Il ne parlait jamais de lui-même qu à la $3^{\circ}$ personne, ne montrait ses effets et instruments que le moins possible et alors même affectait de les regarder comme des talismans' que lui ou son fils pouvaient seuls toucher. Quand il avait guéri quelqu'un il l'envoyait à son marabout ${ }^{22}$ en lui ordonnant de se purifier et de lui faire un présent. Il visitait tous les marabouts et les traitait d'égal à égal, ne faisait pas difficulté de manger devant le peuple, mais faisait toutes ses préparations, prières et incantations pour fabriquer des talismans, en secret. Sa boussole ${ }^{23}$ qu'il portait sur le cœur, son autre boussole de Kater qu'il tenait toujours au côté étaient avec sa montre qu'il ne découvrait jamais ses grandes amulettes ${ }^{24}$. S'il les consultait c'était à son dire pour se mettre en communication avec l'âme de son père ou avec son ange gardien ou bon génie"s. Ses divers voyages étaient autant de vœux ${ }^{26}$ faits pour la guérison opérée ; ses voeux se faisaient à haute voix devant le peuple. Il étudiait la médecine du pays et en suivait parfois les pratiques : les siennes consistaient en écorces de fruits secs et pulvérisés et autres substances innocentes qui acquéraient des vertus par l'occasion et le donneur. Il les délivrait en petite quantité pour favoriser l'opinion qu'il les portait avec lui. Ses remèdes ordinaires étaient la prière à haute voix en langue inconnue, l'imposition des mains, la prescription de certaines pratiques ou de justice ou d'abstinence et quand il s'agissait de la guérison d' un Chek ${ }^{27}$ ou autre personnage il s'imposait (f. 65v) par voeu une privation bien évidente pour tous comme de rester debout toute la journée sans manger. Sur la place, sans boire etc. Son fils le servait comme un esclave, restait debout devant lui tandis qu'il mangeait et observait comme lui une gravité obstinée.

Il se faisait payer de tous, à moins que le malade ne déclarât qu'il n'avait pas de quoi. Auquel cas il donnait ses soins gratis, mais il était très sobre de ces dons parce qu'il en aurait été plus tourmenté. Aux heures consacrées à ces observations il était tout à fait invisible ${ }^{\imath 2}$.

Ses charmes ${ }^{29}$ étaient des inscriptions latines indiquant sa place, sa santé et ses projets. Il partit avec la résolution de ne plus repasser par les mêmes lieux tant parce qu' il n' $y$ avait rien à y apprendre ${ }^{\text {in }}$ que pour ne pas rencontrer ceux que ses charmes $n$ 'avaient pas guéris ou contentés. Il portait l'habit de chaque pays mais beau et distingue $e^{31}$ et ne disait qu aux chefs comme une haute confidence et un secret qui méritait son prix, sa nation et sa foi. Tous les soirs lui et son fils à deux genoux priaient le dieu des chrétiens et la Vierge Marie de sourire à son expédition et de le ramener au seuil de ses pères.

(f.65v) Ses instruments étaient dans une malle de cuir où chacun avait sa case de sore qu' on voyait d'un coup d'oeil s'il manquait quelque chose. Sa marchandise pour les Pays des Kaffirs" étaient des aiguilles que son fils lui donnait comme échange $:$ il se serait cru abaissé par là. Dans sa ceinture comme aussi dans sa poche secrète il portait environ $6.000 \mathrm{~F}$ en or de toutes grandeurs et,nations. Il avait projet de cacher quelques pièces de temps en temps dans des ruines ${ }^{33}$ et de les y faire prendre par un 
chef auquel il aurait besoin de donner quelque récompense. Il se faisait avertir de ces trouvailles par des songes et ne manquait pas de demander sa part du magot.

Quand par une suite de guérisons il avait une surabondance d'objets de valeur inférieure il les faisait échanger par son fils contre des chameaux, chevaux, couvertures en laine et autres objets utiles en voyage. Il avait deux nègres forts, un peu bêtes, hypnophanes ${ }^{34}$ si cela était possible et qui ne le touchaient jamais. Il (f. 66) essayait toujours de les prendre eux et ses autres domestiques parmi les esclaves indigènes du pays qu'il allait visiter. ${ }^{3 s}$ Il ne faisait de présents que lorsqu'il avait reçu trop de choses: quand il n'avait pas reçu assez il ne se faisait pas scrupule de demander. Autant que possible il ne mangeait pas de viande, et demandait partout du lait ${ }^{26}$ soit halyb ${ }^{37}$ soit leban ${ }^{3 *}$. C'était un des meilleurs présents à lui faire. S'il était poussé à bout, mais seulement Jorsque sa vie était en danger il faisait des imprécations terribles.

De temps en temps, il donnait en très petite quantité de ces remèdes héroïques ${ }^{2 n}$ quj produisent un effet évident ; par là fortifiant la foi en ses orviétans $s^{a n}$.

Il écrivait toujours dans le plus grand secret et dessinait de mémoire ou quelquefois avec la caméra lúcida ${ }^{41}:$ jamais en face. Si on lui demandait pourquoi il ne se faisait pas musulman, il répondait : s`il est écrit, il en sera ainsi : je suis un esclave de Dieu : notre Dieu et le vôtre sont un. Il se disait partout un basque petite nation ${ }^{4}$ indépendante entre France et Espagne. ${ }^{33}$

Lorsqu' on le pressait de questions, il disait que ses instruments d'astronomie ${ }^{44}$ servaient à voir dans son pays, que ses amis à des heures fixes regardaient la même étoile que lui : que ses instruments étaient faits pour son village et ne pourraient servir à d'autres qu’à lui, mais que si eux voulaient aller en son pays, il leur ferait voir leurs tentes. Quand il était obligé de montrer de l'or, il disait ne pouvoir en faire que sous certains aspects des astres ${ }^{+5}$ et il expliquait $\operatorname{ces}^{46}$ aspects à sa guise. Avant de quitter une ville, s'il y avait gagné assez d'argent, il faisait tuer un ou deux moutons ${ }^{47}$ ou bœufs et les faisait distribuer aux pauvres.

Quand il voulait passer d'une nation dans une autre il faisait dire par ses messagers que si l'on voulait recevoir ses soins et ses tabus ${ }^{48}$, on ait à lui envoyer une escorte, etc.

\section{Annexe 4}

\section{Juan de Toledo ${ }^{49}$ : pratique d'un magicien morisque (origine musulmane)}

\section{procès de l'Inquisition de Tolède (Espagne), XVII' siècle}

El doctor Juan de Toledo, médico morisco de los antiguos, de edad de beinticinco años, vecino de Toledo, fue testificado por catorce testigos, ocho barones, el uno menor, seis mugeres ; las quatro menores. Seis contestaron los demás singulares de que dos o tres beces, en dos casas diferentes, siendo consultado sobre un hurto que se habia hecho a cierta persona, respondió que alçarian figuras y lo diría. Y preguntado a una mujer si era doncella y respondiendo que si, la escrivió unas letras en la frente y trajeron bufete y derramó encima del la tinta e hizo una forma de espexo redondo y dijo a la dicha muger que se pusiese el rostro encima por la potestad y virginidad de Nuestra Señora y por la del señor San Juan Bautista y de la Santísima Trinidad que me digas verdad en todo lo que te preguntare y digas quién ha hecho el dicho hurto. Y luego la dicha mujer dixo que había visto salir en la dicha tinta a una figura de mujer que le pareció era una de quien sospechaban había hecho el dicho hurto. Y que la dicha figura abía hecho con la cabeça señas diciéndole no. Y luego, el dicho doctor Toledo 
volvió a decir las dichas palabras e hizo salir a otra figura que dijo era la compañera de aquella muger primera y la preguntó si sabía algo de aquel y dio de cabeza que no y el suso dicho, esperança en Dios. Y la dicha muger dixo había visto sobre la dicha tinta, dichas las dichas palabras, salir cinco hombres con cuellos escarolados y otros con balonas. Y preguntándoles el dicho doctor si pareciera el hurto, unos respondieron que sí y los otros que no. Y que no pareció ninguna cosa de las hurtadas. Y que también hacía la dicha figura de la tinta para sacar tesoros. Y que tenía el libro de San Cipriano y dio el de la Picatrix a cierta persona para sacar tesoros. Y que le habían traydo de Madrid para que descubriese otro hurto. Y dio un papel a otro hombre en que después de haber alçado figura decía el nonbre de los ladrones. Y habiendo faltado unos corales a esta muger, consultándolo con el suso dicho otro día, le dio las señas de las personas que los habían hurtado y las de la casa en que se hallarían, donde se hallaron en poder de la persona en quien concurrían las dichas señas. Decía publicamente que lo hacía con licencia de los inquisidores.

Y siendo consultado para descubrir otro hurto dixo donde se hallaría y ansi pareció. Y para descubrir otro hurto hizo las dichas señales de la tinta en un banco y escribió a un muchacho en la frente unas letras diciendo las dichas palabras de Nuestra Señora y San Juan. Y diciendo el muchacho que no había visto nada, el dicho doctor Toledo bolví́ a decir « sal por tu grande amigo Barrabás ». Y llamó a otros muchos demonios por otras beces. Y llegó el dicho muchacho, bio su rostro y el del dicho Toledo y el de otra persona en la dicha tinta. Y para ber cierta persona que estaba en las Indias hiço la dicha figura. Y se le vio estar sentado con un frayle en un banco.

Calificóse que de más de incurrir en las censuras del motivo propio de Sixto Quinto contra los iudiciarios que tratan de adevinar las cosas que prenden del libre albedrío. el hecho es supersticioso, donde se mezclan palabras sagradas con profanas. $\mathrm{Y}$ es sospechoso en la fe. Con esta testificación fue preso en las cárcelas secretas deste Santo Oficio y mandado hacer causa con él.

\section{Annexe 5}

\section{La poupée de cire et le magicien maure ${ }^{s_{0}}$ : fiction du XVII' siècle}

Sintió tanto el mal aconsejado mozo esto, que como desesperado, con mortales bascas, fue a su casa, dondo estuvo muchos días en la cama con una enfermedad peligrosa. acompañada de tan cruel melancolía que parecía querérsele acabar la vida y viéndose morir de pena, habiendo oído decir que en la ciudad había un Moro, gran hechicero y nigromántico, le hizo buscar y que se le trajesen para obligar con encantos y hechicerías a que le quisiese doña Inés. Hallado el Moro y traído se encerró con él, dándole larga cuenta de sus amores, tan desdichados como atrevidos, pidiéndole remedio contra el desamor y el desprecio que hacía de él su dama, tan hermosa como ingrata. El nigromántico agareno le prometió que dentro de tres días le daría con que la dama se le viniese a su poder, como lo hizo". Que como ajenos de nuestra fe católica no les es dificultoso, con premios que hacen al demonio, aun en cosas de más calidad. lograr estas cosas. porque pasados tres días vino y le trajo una imagen de la misma figura y rostro de doña Inés. que por sus artes la había copiado del natural como si la tuviera presente. Tenía en el remate del tocado una vela de la medida y proporción de una bujía de un cuarterón de cera-verde ; la figura de doña Inés estaba desnuda y las manos puestas sobre el corazón, que estaba descubierto, clavado en él un alfiler grande, dorado. a modo de saeta, porque en lugar de la cabeza tenía una forma de pluma del mismo metal y parecía que la dama quería sacarle con las manos que tenía encaminadas a él. 
Díjole el Moro que, en estando solo, pusiese aquella figura sobre un bufete y que encendiese la vela que estaba sobre la cabeza, que sin falta ninguna vendría luego la dama y que estaría el tiempo que él quisiese mientras él no le dijese que se fuese y que cuando la enviase no matase la vela, que en estando la dama en casa ella se moriría por sí misma, que si la mataba antes de que ella se apagase corría riesgo la vida de su dama ; y asimismo que no tuviese miedo de que la vela se acabase aunque ardiese un año entero, porque estaba formada por tal arte que duraría eternamente mientras que en la noche del Bautista ${ }^{52}$ no la echase en una hoguera bien encendida. Don Diego, aunque no muy seguro de que fuera verdad lo que el Moro le aseguraba, quedó contentísimo, cuando no por las esperanzas que tenía, por ver en la figura el natural retrato de su natural enemiga, con tanta perfección y tantos colores que, si como no era de más altor de media vara fuera de la altura de una mujer, creo que con ella olvidara el natural original de doña Inés, a imitación del que se enamoró de otra pintura y de un árbol. Pagóle al Moro a su gusto el trabajo y despedido de él aguardaba la noche como si esperara la vida; y todo el tiempo que venía se dilató en tanto que se recogía la gente, una hermana suya viuda que tenía en casa y le asistía a su regalo y se le hacía una eternidad. Tal era el deseo que tenía de experimentar el encanto, pues recogida la gente, él se desnudó para acostarse y dejando la puerta de la sala nada más apretada, que así se lo advirtió el Moro, porque las de la calle nunca se cerraban por haber en la casa más vecindad, encendió la vela y poniéndola sobre el bufete se acostó, contemplando a la luz que daba la belleza del retrato. Y cuando la vela empezó a arder, la descuida doña Inés, que estaba ya acostada y su casa y gente recogida porque su marido aún no había vuelto de Sevilla por haberse recrecido a sus cobranzas algunos pleitos, privada con la fuerza del encanto y de la vela que ardía de su juicio y, en fin, forzada de algún espíritu diabólico que gobernaba aquello, se levantó de su cama y poniéndose unos zapatos que tenía junto a ella y un faldellín que estaba con sus vestidos sobre un taburete, tomó la llave que tenía debajo de su cabecera y saliendo fuera abrió la puerta del cuarto y juntándola en saliendo y mal torciendo la llave se salió a la calle y fue en casa de don Diego, que viendo un caso tan maravilloso quedó fuera de sí...

\section{Annexe 6}

\section{L'archevêque de Samos : un magicien grec de fiction et son page} 1694

El arzobispo de Samos camina a grandes trancos por la celda del convento de Santo Domingo que le sirve de prisión. Walter ha escapado llevándose lo único que al griego le quedaba : su grueso anillo de oro cuya esmeralda ostenta labrado el mochuelo grato a Minerva. Mientras va y viene, colérico, el arzobispo se tortura pensando cómo habrá conseguido robarle la sortija. Los dedos de su mano derecha acarician incesantemente el anular izquierdo, como si esa fricción mecánica pudiera hacer surgir, bajo las yemas, la lisura familiar de la piedra preciosa.

Walter, su paje, desapareció hace quince horas con el anillo. ¡El anillo merced al cual Fray Joseph Georgerini proyectaba comprar su evasión a los carceleros ! Y ahora no le resta nada, ni siquiera la esperanza. El también parece un mochuelo, un enorme mochuelo absurdo, con sus ojos redondos y crueles, su nariz corva, su cara amarilla y sus ropas talares pardas y sucias, cuyas mangas flotantes se agitan como alas en la celda conventual.

¡Adiós, pues, a los planes de huida ! El gobernador del Río de la Plata, el obispo y los comisarios de Ia Inquisición se salieron con Ja suya, y el arzobispo de Samos 
deberá emprender el camino de Lima, donde le aguardan los señores del Santo Oficio. Todo por culpa de Walter, por culpa de ese condenado inglés, hijo de mala madre, que sin duda se estará riendo ahora, rumbo al norte o al sur, lejos de los dominicos, de los interrogatorios, de los tribunales del Perú.

Fray Joseph Georgerini no teme el encuentro con los inquisidores. Otros casos más graves ha soportado en el curso de su vida azarosa y es hombre de mucho ingenio para que puedan hacerle trastrabillar las zancadillas de unos pequeños eclesiásticos coloniales. En cambio Walter sí lo temia. ¡Había que verle palidecer y demudarse, cuando se hablaba de los tormentos! Pero el arzobispo, con toda su ciencia sutil, no quiere enfrentarse con los agentes romanos. Prefiere que en Roma olviden su existencia. Y va y viene, rabioso, en tanto que las campanas del convento llaman a la primera misa.

Su cólera sube de punto. Hay en sus ojos de mochuelo un brillo peligroso. Desata el largo cordón que le ciñe la cintura y lo coloca en el piso, dibujando un triángulo. Luego se descalza, penetra en él, alza las palmas y comienza su invocación, implorando a Satán, Leviatán, Elioni, Astarot, Baalberit.

Las fórmulas mágicas de los grimorios, las del Libro de San Cipriano, las de la Clavícula de Salomón, resuenan en la celda de Buenos Aires. El arzobispo de Samos es ante todo un hechicero. Ahora no semeja un mochuelo sino un macho cabrío, temblorosa la barba, las cejas juntas, revuelto el pelo como una cornamenta, mientras repite por lo bajo los conjuros que otorgan la alianza del Demonio:

- Belfegor, Tanín, Belial, Alastor, Baal...

Hace más de cuatro meses que la impaciencia roe a Fray Joseph en el convento de Santo Domingo. Sólo una degraciada tempestad y el naufragio de su barco pudo llevarle a playa de tanta miseria. Cuando le condujeron desde las trojas, medio muerto, sobre una parihuela, entreabrió los párpados y vio desfilar a su lado la infinita pobreza de Buenos Aires que clamaba por la lluvia. Seguían su camilla, haciendo ademanes de teatro, el gobemador don Francisco de Robles, tres o cuatro oficiales reales, algunos soldados, y el extraño séquito del arzobispo : un paje inglés y los dos frailes agustinos, un español y un napolitano, que había recogido en sus andanzas. Detrás zozobraban los restos del navío y el lento olear del Río de la Plata batía contra unos pocos cajones tumbados en el barro. Todo se había perdido.

Días complicados aguardaban al griego. Naturalmente, nadie comprendía quién era. El comisario porteño del Santo Oficio estudió sus papeles dándose aires de sabiduría, pero fue en vano. Dio vueltas y vueltas a las dos bulas escritas con letras ilegibles sobre pergaminos en forma de media luna, por medio de las cuales el Gran Turco despojaba a Fray Joseph del arzobispado de la Isla de Samos. El comisario resoplaba sobre las iniciales de oro, fruncía las narices y aspiraba el sospechoso olor de la herejía.

Los dos agustinos salvaron su responsabilidad de inmediato. Arguyeron que le habían conocido recientemente, y abriendo las manos en abanico sobre la boca, apuntaron que se dedicaban a ordenar sacerdotes cobrando por ello buenas monedas de plata. El arzobispo mascullaba en griego, en latin, en turco, barajando los vocablos. Su criado sólo hablada inglés. Era imposible comprenderles y ellos mismos alimentaban la confusión adrede con tanto sonido ronco. Pero el comisario inquisitorial buscaba su pista dentro del laberinto. Iba arremangado, congestionado, repitiendo las preguntas.

Unas veces creía deducir que Fray Joseph Georgerini, griego de nación, cismático que había abjurado, había obtenido el permiso del Papa Clemente X para decir misa según el rito griego. Deducía otras que el forastero había vagado de Santos a Roma y 
de alli a Florencia, a París, a Marsella, a Londres, a Lisboa : que los ingleses le habían reducido a prisión ; que había ambulado por Madrid con el hábito desgarrado, como un profeta antiguo. Y por fin, cuando estaba seguro de que Su Majestad vería con agrado que las autoridades de Buenos Aires procedieran contra el intruso, el griego sagaz lograba torcer el expediente y dar la impresión de que el monarca le había autorizado a pedir lismona en sus dominios por tiempo de un año.

El comisario don San Sebastián Crespo y Flores se mesaba los escasos mechones y volvía a empezar. Desesperado, cerró las carpetas, escribió al Santo Oficio de Lima y ordenó que el arzobispo de Samos, o lo que fuera la jerarquía de su obligado visitante, se encerrara bajo custodia en el convento de los hijos de Guzmán. Allá se fue Fray Joseph Georgerini, aleteantes las mangas de su ropón. Le quedaba su esmeralda, con el grabado mochuelo de las viejas monedas atenienses. Seduciría con ella a sus guardianes y se podría escapar. Y ahora Walter se la había robado...

- Satán, Leviatán, Elioni, Astarot, Baalberit...

El arzobispo de Samos Ilama al Demonío en su socorro ; al Diablo a quien Erasmo vio en las pulgas de Rotterdam, a quien el ermitaño San Antonio escupió en la cara, a quien el Papa San Silvestre metió en una cueva ; a quien San Cipriano abrazó en su juventud. $Y$ la celda se ilumina con el color del azufre en tomo al brujo que semeja un macho cabrío.

Walter escapa a uña de caballo camino de Córdoba. En su anular izquierdo fulge la esmeralda con la pequeña ave rapaz que se nutre de reptiles y de roedores. El paje se detiene a pasar la noche bajo un espinillo. En la soledad que le oprime y le asusta, piensa, para distraerse y darse ánimo, en el mal rato que estará viviendo su señor. Una leve sonrisa asoma a sus labios pero pronto se borra. La imagen iracunda del arzobispo de Samos, a quien supone entregado a quién sabe qué ritos nefandos en el convento de Buenos Aires, basta para invadirle de zozobra. Durante los últimos meses, en el barco, ha espiado a Fray Joseph en más de una ocasión por el ojo de la cerradura, y le ha visto modelando figurillas de cera en las que luego clavaba alfileres agudos, o inclinado con el rostro descompuesto sobre sus libros arábigos. Pero por más que el griego quiera, no podrá alcanzarle hasta aquí con sus mañas. Hay muchas leguas entre ambos. El muchacho enciende una fogata y se tumba a dormir arrebujado. Allá cerca, su caballo pasta en los cardales. La noche pesa encima con su terrible negrura.

Horas más tarde, Walter despierta bruscamente. Algo, quizás un insecto, le ha picado el anular, en la mano donde lleva la sortija. La hoguera se ha extinguido casi y al tímido resplandor de las brasas el paje distingue, sobre el anillo, en la segunda falange, una diminuta mancha azul. Alrededor la noche acentúa su desamparo. La pampa no goza ni de una estrella ni de un grillo. Sólo rompe el silencio, en las tinieblas cercanas, el balido de un animal que podría ser una cabra salvaje. Temeroso. Walter silba a su caballo y como respuestra oye, dentro de la fronda del árbol que le cobija, el grito de un ave oculta, quizás mochuelo, como una risa falsa, destemplada. El muchado se esfuerza por divisar algo en la sombra, pero la masa del espinillo se funde con las tintas noctumas. Se pone de pie y, extendiendo los brazos como un ciego. sale en busca de su alazán. Lo silba de nuevo, lo llama, y entretanto el balido responde a la risa agorera que parte del tronco. El dedo le quema como si lo hubiera arrimado al rescoldo. Acaso le haya mordido una víbora. Lívido de terror, Walter se quita la esmeralda y la coloca en el anular derecho. Mientras las palpa con angustia, siente que las falanges izquierdas se le hinchan y que el dolor se insinúa hacia la muñeca, hacia el brazo. Entre los cardos roza por casualidad un fragmento de la brida de su cabal- 
gadura. Se oprime la mano envenenada que al tacto parece algo monstruosa y caliente. A la distancia, escucha el alegre relincho del caballo que huye por la llanura desierta. Walter regresa a tropezones al refugio del fuego. Ahora le arde la otra mano, el otro anular, a la altura de la sortija. Saca también de allí el anillo y lo desliza en su faltriquera. Acerca los dedos a las brasas y ve que los de una mano, tumefactos, agarrotados, con las uñas irreconocibles, comienzan a cubrirse de una transpiración verde y mohosa. En la otra ya avanza la gangrena azul.

El paje llora de miedo y se echa a correr entre las matas. Bajo su faltriquera, en el muslo, la misma mordedura le irrita la came. Toma el anillo griego, el anillo maldito, y lo arroja a la noche. La piedra brilla en el aire como un carbunclo. Pronto, los brazos de Walter penden, inertes, perdidos. Arrastra la piema que le pesa como si fuera de plomo. Comprende que no podrá continuar así por mucho tiempo. Y en tanto vaga cayendo y levantándose, por el llano cruel, gimiendo como un loco, le persigue la risa del mochuelo invisible, que revolotea en la oscuridad y le golpea la cara con las alas duras."

Manuel Mújica Laínez, Misteriosa Buenos Aires, Seix Barral, 1985

\title{
Yvette CARDAILLAC-HERMOSILLA
}

\author{
Université de Bordeaux III
}

\section{NOTES}

1. Sánchcz Lavcga. Agustín. "Planeta en el universo". Congrds International pour le centenaire d"Antninc d'Abbadic. 1997-1998. Eusko Ikaskuntza, Faculté Pluridisciplinaire de Bayonne. 1997, (sous pressc).

2. Cardaillac, Yvette "L'orientalisme au châtcau d'Abbadie". Congrès Intemational pour le centenairc d'Antoinc d'Abbadic. 1997-1998, Eusko Ikaskuntza. Faculte Pluridisciplinaire de Bayonne, 1997, (sous prosse).

3. Chiromancic : du grec kheir. kheiros : main ct mantcia : divination, art de deviner el de predire par linspection des lignes de la main.

4. Chirognomonic : art de connaiture le caractère des personnes par la forme de la main.

5. p. 105.

6. Jamet. Christian. Delacroix, images de / Orienu. Paris. Herscher. 1995, 64. "Le combat du giaour ct du pacha". 1826. Art Institute of Chicago, tableau d'après le poc̀me de Byron où I'on observe la mélce furicusc des hommes et des animaux combattants selon la tragique histoire de Leila jeté a la mer par le Turc Hassan (ici sur un cheval blanc), p.30. Une nouvelie version est peinte en 1935, p. 31, elle est plus précise. on distingue le visage des cavaliers car le peintre a sćjourné en Orient.

7. Urkizu Patri, Arkotxa-Searcia Aurelia, Arkotxa Fermin. Pensée, études et vovages de 1835. II. Antoinc d'Abbadic 1810-1897. Congrès International de Hendaye, San Sébastien, Eusko Ikaskuntza, 1997, 160 p.p. 123-125.

8. L'alchimic cst une des "sciences" traditionnclles de I'Islam désignée par le même terme que la chimic proprement dite. Al-Kimya en arabc.

Thoraval Yves. Dictionnaire de civilisation nusulmane, Paris, Larousse, 1995. 332 p.p. 11.

9. Amulettc : du latin amulctum. Certaines amulettes sont utilisées contre le mauvais ail et constitucnt un des éléments essenticls de la magic prophylactique. La main de Fatima est un symbole utilisé de l'Afnique du Nord a I'Inde dans cet objectif. On porte aussi divers objets (coquilles de mer. comes de corail. crocs de sanglicr. pcaux de couleuvre) pour sc protéger des caprices des génies. $318 \mathrm{p}$

10. Cohn. Norman. Démonolatrie et sorcellerie au Moven Age. fantasmes et réalités. Paris. Payot. 1982.

11. Heningsen. Gustav. The witche s advocat : Basque witchcraf and spanish Inquisition (1609-1614). University of Nevada. 1980, El mayor proceso de la Historia, Navarra 1609-1614), in Historia 16. $n^{\circ} 80$. annec VII. p. 46-54.

12. P. $59:$ créancc / p. $76:$ Doyle / p. 77 : intendance / dunvie bail / Walsh arrieré / p. $78:$ Mme Dunnc/ P 79 : comples / P. 80 Dublin 21 févricr / P. 105 leure à Daguerre / P 109 deue / P. 117-118: livres à relier 
/ P 120-121/P. 122 : Irais de toiture / P. 124.

13. Voir la représentation d'Antoine d'Abbadie en togc et turban éthopien accompagné par son esclave Abdullah.

14. Musset. Alfred, de, Namouna, XXIV, 1832

15. Exolisme: mot qui vient du grec.

16. Urkizu Patri, Arkotxa-Searcia Aurclia, Arkotxa Fermín. Pensée, études et voyages de 1835, II Antoinc d'Abbadic 1810-1897. Congrès Intcrnational d'Hendayc, San Sébastien, Eusko ikaskuntza, 1997, 160 p.p. 55 , foljo 15.

17. Abbadic. Antoine, d'. Pensée, études et vovages de 1835, édition de Patri Urkizu, avec la collaboration de Aurelia Arkotxa-Scarcia et Fermin Arkotxa, San Sebastien, Eusko lkaskuntza, 1997, 160, p. 105.

18. Kaipha : Haîfa (Syrie), ville du desen au pied du mont Carmel sur la baie d'Acre, port important, plaine ferile aux environs. Nous ne sommes pas en Arabie, lieu d'origine de la poésie d'amour qui scmble avoir inspiré ics troubadours. Nous constatons plutôt la reprise diimages traditionnelles du chef dans lc désert et du thème de l'amour courtois qui reprend vigueur avec le style troubadour remis en vogue par les romantiques.

Par ailleurs "partant pour la Syrie" est le tutre d'une romance de 1810 auribué à la Reine Hortensc et dont le veritable autcur serait le Comte Alex de Laborde, archéologue et membre de l'Institut, avec une musique probable du flûtiste Philippe Drouet, secrétaire musical de la reine. Ce chant devint la marche officielle de toutes les fêtes du second empire.

19. Simoun : de l'arabe samoûn, vent violent extrêmement chaud et sec qui souffle sur les régions déscrtiques de l'Arabie, de la perse el du Sahara, mot qui apparaît dans la première moitié du XIX' sičclc en France. Voir les informations donnes par Antoine d'Abbadie dans l'article d'A. Arkotxa Scarcia : "L'Orient comme virtualité dans le camet de 1835 d'Antoine d'Abbadie".

20. Urkizu Patri, Arkotxa-Searcia Aurelia, Arkolxa Fermin, Pensé, éludes ef vovages de 1835. 11. Antoine d'Abbadie 1810-1897, Congrès International d'Hendaye, San Sébastien, Eusko Ikaskuntza, 1997. 160 p.p. $123-125$.

21. Talisman : voir deuxièmc page du commentaire.

22. Marabout : musulman pieux, sanctifié par une vie ascétique et exemplaire contemplative vivant près d'une petite mosquéc qu'il dessert. Il lutte contre les passions de la chair pour arriver à l'union avec Allah. Mystique. il a des extases qui lui dévoilent les secrets du monde intangible. La désobéissance à ses ordres est un crime inexpiable qui entraine les châtiments infemaux. Son influence est grande en Afrique du Nord. II fait le plus souvent partie d'une confrérie hiérarchisee. Parfois ils deviennent des saints personnages. d'autres fois des charlatans utilisent ce terme pour tirer partie de la confiance des gens.

23. Boussole : la boussole fonctionne à partir de l'aimant. La pierre aimantée par ses propriétés remarquables pour celui qui ne connait pas le mécanismc était utilisée au XVII' sjècle dans les pratiques magiques. en particulier on la portait comme talisman au même titre que la pierre d autel, qualites et origines qui leur conféraicnt un caractčre magique ou sacté.

24. Objet porte-bonheur; nous retrouvons le scientifique qui a besoin pour ses voyages de boussole et de montre.

25. Génie (bon) ange gardien. A l'origine en Arabic, les bédouins polythêistes avant l'Islam adoraicnt de nombrcux dieux et craignaient les génies invisibles issus du feu, Ics Djinns, certains étaient bons, d'autres mauvais. Ils s'organisaient en sociétés sur le modèle humain. Dans le texte de génie, l'âme du pèrc ct l'ange gardien sont mis en parallèle, le demier terme mettant l'accent sur la bonté de ses entités.

26. Vœu : du latin votum. promesse faite a la diviníle, d'accomplir un acte ou de s'en absicnir. Résolution que l'on prend et que l'on veut rcgarder comme sacrée. celte pratique courante dans lc monde musulman s'accompagne d'une visite chez le marabout. Aux grilles de la petite mosquée, les fideles attachent des foulards qu'ils nouent dans le coin en témoignage de leur vou ou de leur demande. lls viennent le dénouer apres rtalisation. Comme trace matérielle on observe aussi l'application de mains cnduites de henné sur les portes ou sur les murs.

27. Chcik ou Scheik : de l'arahe scheikh, vieillard, chef de tribu.

Cheik el islam : le grand mufti

Cheik cl haram : le gouvemeur de Médine, gardien du tombeau de Mahomet.

28. Invisible : ambiguité du mot ici invisible pour garantir la concentration scientifique.

29. Charmc : sens partiel, assimilé ici au carnet de voyage, ecrit en latin, mise en abyme du texte avec lc systeme des poupces russes:

1. Le grand texte (macro texte) (carnet de voyage)

2. Le textc intermédiaire ou portrait du magicien

3. Le charme ou talisman (le micto texte) en latin indiquant la place, la santé et les projets écho du macro texte.

Charme : du latin carmen, mot magique, enchantement magique ; rompre le charme. Ce qui produit cet cnchantement : acheter un charme. sieccle

30. La soif de savoir passe avant Ies relations humaines, attitude caractéristique des énudits du XIX

31. L'allure digne ct noble caractérise le personnage.

32. Tcrme péjoratif d'origine arabc utilisé pour désigner les infidèles, peut-être formé sur Kaffa. 
Kaffa (Caffá) : Province du Sud-Ouest de l'Ethiopie limitée au Sud-Ouest par le Soudan, elle occupe la vallce du côté droit de la rivière Omo. Son relief est montagneux ( $2413 \mathrm{~m}$ ).

33. Trésor dans les ruines : la croyance aux trésors souterrains remonte à l'Antiquité, on les croyait protégés par des génies qui les dérobaient aux recherches des humains. On pensait que lorsqu'on trébuchait de façon répété à un endroit précis, là se trouvait un trésor enterré. En fait, la coutume d'enterrer les morts avec leurs bijoux ct parfois les effets nécessaires au voyage dans l'au-delà surtout Jorsqu'il s'agissait de personnalités donnait lieu à la découverte dans les ruines de trésors divers (pyramides, tombeaux romains). En Espagne on pensait que les Maures à leur depart avaient cnterre leurs biens.

34. Hypnophane : qui parle dans son sommeil.

35. Abdullah fut l'esclave éthiopien d'Antoine d'Abbadie, ramené en France au retour de voyage et représenté avec son maître en habit du pays. toges et turbans.

36. Lait : les hommes dans la prison de Cook font deux repas avec du pain et du lait, p. 75 du carnet de voyage. Antoine d'Abbadie observe que ce régime tout simple les engraisse beaucoup et qu'ils sont en bonne santé (un seul est mort depuis dix ans).

37. Halyb : lait frais, information donnte par Bianca María Scarcia-Amoretti, professeur du Département d'Etudes Orientales de l'Université la Sapienza de Rome.

38. Leban : lait caillé, information donnée par Bianca María Scarcia-Amoretti, professeur du Département d'Etudes Orientales de l'Université de Sapienza de Rome.

39. Dans le camet de voyage nous constatons l'intérêt pour les premicts soins à donner en cas de maladie. l'auteur les note consciencieusement.

40. Orwiçans : it. orvictano. Electuaire (remède d'une consistance de pâte molle, poudre mélangée dans un sirop ou dans du miel) inventé par Ferrante d'Orvieto et en grande vogue a Paris au XVI' siècle. Droguedc charlatan. Fig. : marchand d'Orviétan = charlatan

41. Caméra lúcida : caméra : nom latin de la chambre claire pour dessiner. Vermeer l'utilisait.

42. Au masculin dans lc manuscrit sans doute du fait que l'adjectif en anglais est invariable.

43. References autobiographiques évidentes : Antoine d'Abbadie est connu pour ses travaux d'astronomie.

44. Références autobiographiques évidentes : idem.

45. Allusions à la recherche des alchimistes (Al-chime) ; al : article arabe, transformation recherchée ou symbolique des corps en or.

46. Ces: dans le texte ses, faute d'orthographe évidente par le sens que nous rectifions ici.

47. L'Aid El Kebir est une des grandes fêtes musulmanes où le sactifice du mouton que l'on partage avec les pauvies rappclle celui d'Abraham.

48. Tabu pour tabou : il existe des objets pouvant devenir tabous par la volonté de celui qui les possede. Les chef́s. les prêtres, les magiciens. les cadavres sont tabous par naturc. Cet état d'essence religieuse montre le respect mêle de terreur qu' inspirent aux peuples non civilisés les actes principaux de la vie. Les phénomènes de la génćration, de la naissance et de la mort. les personnes investies de l'autorité ou de pouvoirs surnaturels. Le magicien veut garder ses distances et son mystcre.

Au Moyen Agc, l'astronomic se développa grâce aux travaux de savants arabes comme Al Biruni : Ic perfectionnement de l'astrolabe. permit de mesurer la position de la terre, face aux astres à partir de la ligne dc l'horizon. Par ses travaux. Antoine d'Abbadic contribue aux progrès de cette science. La boussole, invention chinois. fut transmise a l'Europe.

49. Archives Historiques Nationales. Inquisition de Tolede, relation de cause $2106 / 9$ de 1610 , Juan de Toledo. médecin morisque.

50. Zayas. María de, Novelas ejemplares y amorosas, Madrid, Alianza Editorial, 1990, 226 p. "La inocencia castigada" p. 140-143.

La figura de cera cs de uso corriente para prácticas mágicas en toda Europa Sin embargo, figura de forma excepcional en los procesos de Inquisición en Espana por la influencia musulmana que rechaza las represcntaciones humanas. Se utilizan más bien bolas de cera o siluetas recortadas. María de Zayas aplica lors esquemas tradicionales de los critianos a un Moro y de hecho no le corresponde por su cultura.

51. La magie amoureuse est cclle qui est la plus présente dans la fiction littéraire, elle apparaît aussi dans les procès d' Inquisition des vieux chretiens mais elle est pratiquement inexistante dans ceux concernant Ics dernicts musulmans d'Espagne (XVI", XVIle sieclic).

52. La nuit de Saint-Jcan, 24 juin, est par tradition propice aux pratiques magiques.

53. Le portrait agissant du magicien constitue un morceau de bravoure dans la litterature classique, moderne ct contemporaine mais aussi dans les documents juridiques : l'un expliquant sans doute l'autre. Genre litteraire mincur, il peut s' inclure dans les scènes de genre dont le traitement varie selon les modes, ic contcxtc historique et géographique. 